\title{
Ripresa della tradizione e innovazione compositiva: la medicina nei Posthomerica di Quinto Smirneo*
}

\author{
LEYLA OZBEK
}

L'interesse di Quinto Smirneo per i temi medici è senza dubbio una delle più evidenti peculiarità dei Posthomerica: l'analisi di questi punti - descrizioni di ferite o di cure, similitudini riferite a patologie, attenzione ai dati anatomici e fisiologici - mette bene in luce un elemento rilevante della tecnica compositiva di Quinto, ossia la continua tensione tra concezioni mediche e anatomiche della tradizione mitologica precedente, che rappresentano per lui un imprescindibile modello-codice, e allo stesso tempo l'innovativo inserimento, dove la narrazione lo consenta, di dati anatomici e fisiologici più in linea con le conoscenze scientifiche del suo tempo. Scopo di questo lavoro è appunto considerare come Quinto sviluppi, nei vari punti che lo richiedono, questi temi medici, legandosi al sottofondo intertestuale della tradizione precedente e contemporaneamente innovandolo con uno stile così attento a particolari medici da risultare a volte compiaciuto, oppure da scivolare nel macabro.

\section{Variazioni sul tema omerico: le ferite di guerra e le attività dei medici}

Un esempio della tensione di Quinto tra l'adesione al modello-genere omerico e il contemporaneo allontanamento da esso si riscontra all'interno della narrazione di combattimenti, più precisamente nella descrizione di particolari ferite di guerra. È proprio nell'approccio a questo determinato aspetto della narrazione epica che Quinto si confronta maggiormente con il sottofondo intertestuale omerico, dal momento che, se si eccettuano casi eccezionali, il testo di Omero si rapporta con il sapere medico quasi esclusivamente in merito ai traumi ricevuti in battaglia. Se si consultano gli studi sulla medicina omerica, riguardanti soprattutto questo

\footnotetext{
* Ringrazio, oltre a Manuel Baumbach, Silvio Bär e tutti i partecipanti all'Internationale Tagung sui Posthomerica, Glenn W. Most e il Seminario di Ricerca di Filologia Greca della Scuola Normale Superiore di Pisa per i preziosi suggerimenti. Le edizioni da me usate per le opere citate più spesso sono Vian $(1963 ; 1966 ; 1969)$ per i Posthomerica, West $(1998 ; 2000)$ per l'Iliade e Lloyd-Jones / Wilson (1990) per il Filottete di Sofocle.
} 
aspetto, ${ }^{1}$ l'elemento che emerge immediatamente è l'estrema diversificazione delle ferite ricevute in battaglia, anche all'interno di una narrazione formulare come l'epica omerica. In questo caso, la varietà riguarda sia la localizzazione dei colpi, sia l'arma usata sia infine l'esito più o meno fausto sul guerriero ferito. ${ }^{2}$

La grande varietà di ferite presenti nell'epica omerica, che vengono molto spesso riprese da Quinto e su cui non ci soffermeremo perché rappresentano semplicemente un calco del modello precedente, non impedisce tuttavia all'autore dei Posthomerica di variare nella propria composizione dando più spazio, sia per numero di volte in cui è impiegato che per l'aggiunta nella descrizione di dettagli precisi e macabri, a un tipo preciso di ferimento. Si tratta dell'amputazione di una parte del corpo di un guerriero, evento che permette all'autore non solo di descrivere la ferita ma anche, riprendendo e nello stesso tempo ampliando il modello omerico, di indugiare su un particolare non sempre aderente alla verosimiglianza medica ma comunque di grande impatto, ossia particolari attività residue della parte amputata.

Quinto infatti descrive varie e diversificate attività, dopo la recisione, di una parte del corpo (soprattutto degli arti superiori), elemento del tutto assente dalla narrazione omerica. In tutti i casi in cui narra l'amputazione del braccio (o dell'avambraccio), infatti, Omero non cita nessuna ulteriore attività dell'arto amputato, limitandosi al massimo a descriverlo sanguinante a terra. ${ }^{3}$ Quinto,

1 Molti lavori, dai primi ancora ottocenteschi ad alcuni più recenti, si sono concentrati sulla catalogazione a livello statistico delle ferite presenti nella narrazione omerica. In particolare, da ricordare Daremberg (1865) e - con l'inserimento di una tavola sinottica dei risultati - Frölich (1879); l'elenco delle ferite è ripreso anche da Albarracín (1971). La tabella di Frölich è riprodotta da Grmek (1985) 55 ed è in seguito perfezionata da Saunders (2004), che opera tramite l'uso dei recenti programmi informatici un'attenta revisione di tutti i ferimenti e amplia la rosa delle armi utilizzate.

2 Limitandosi a elementi utili per la nostra argomentazione sull'enorme varietà di ferite presenti nell'epica omerica, basti pensare che Daremberg (1865) ha proposto 140 localizzazioni diverse di ferite da combattimento, che sono state aumentate a 147 da Frölich (1879): considerando solo le macrozone all'interno del corpo umano, si possono distinguere colpi alla testa, al collo, al tronco, alle membra superiori e alle membra inferiori, per una gran parte dei quali si può ancora considerare se siano inferti anteriormente, nella zona laterale o posteriormente. A questo si aggiunge la possibilità (ben analizzata da Saunders [2004]) che le ferite siano provocate da un'arma tagliente (spada, lancia, freccia) o contundente (pietra), oppure se l'arma sia brandita (come spada e lancia) o scagliata (frecce, pietre e giavellotti), elemento che rende diverso il meccanismo di trasmissione della forza impiegata per il colpo. Un altro dato fondamentale è se la prognosi sia fausta o meno: riguardo a questo punto, come giustamente notano Grmek (1985) 64-65 e Saunders (2004) 16, l'epica omerica per ragioni di rapidità nello sviluppo della trama comporta solo due possibilità: la morte (immediata o quasi) in seguito alla ferita riportata oppure la completa (e rapida) guarigione con la ripresa delle attività abituali.

3 Quando per esempio Euripilo colpisce alla spalla Ipsenore tagliandogli il braccio, l'arto è descritto solamente cadere a terra insanguinato (Il. 5.80-83). In un altro caso, invece, la narrazione è ancora più stringata e dopo il colpo che recide l'arto non si aggiunge nessun ulteriore dettaglio (5.146-147, Diomede uccide Ipeirone tagliandogli il braccio). Meno chiara è la narra- 
invece, nella descrizione di colpi che recidono un arto superiore aggiunge spesso in modo innovativo il dettaglio macabro delle attività residue dopo l'amputazione, ampliando quindi secondo il gusto dell'orrido diffuso nell'epica tarda (ma non solo) un pattern presente in parte nel modello omerico. ${ }^{4}$ Due descrizioni di attività residue dell'arto amputato si trovano nello stesso libro, l'undicesimo, e sono esse stesse ampliate e modificate, dal momento che non solo presentano attività diverse dell'arto separato dal tronco, ma la prima contiene inoltre una similitudine che accentua l'effetto macabro.

In Q.S. 11.71-78 il braccio, reciso dalla spalla, una volta a terra continua a ripetere l'ultimo movimento che compiva prima di essere amputato, ossia brandire

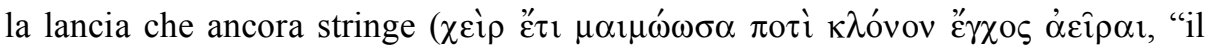
suo braccio che ancora desiderava fortemente brandire la lancia nella mischia",

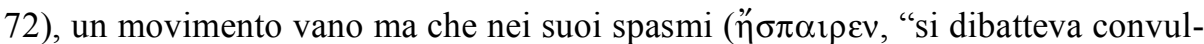
samente", 74) ricorda quello della coda recisa di un serpente che continua a contorcersi senza però riuscire a uccidere l'uomo che l'ha tagliata (74-76). ${ }^{5}$ Dopo questa duplicazione dell'amputazione dell'arto attraverso l'immagine speculare della coda recisa, il narratore chiude l'immagine ritornando al braccio del guerrie-

zione della morte di Mari per mano di Trasimede, il quale prima di ucciderlo gli colpisce l'avambraccio con la lancia (16.321-325), caso che Daremberg (1865) 71 n. 2 inserisce ipoteticamente tra le amputazioni dell'arto superiore.

4 L'amplificazione del racconto tramite l'inserimento di dettagli macabri pare infatti essere una delle note centrali della produzione letteraria tarda: basti pensare con Most (1992), in ambito romano, alle opere di periodo imperiale, come il teatro di Seneca, il Bellum civile lucaneo e anche i Punica di Silio Italico e la Tebaide staziana. In merito tuttavia alla presenza di temi macabri nella letteratura epica latina di periodo imperiale, e più in particolare alle attività residue di parti del corpo amputate, le più interessanti per la nostra indagine per via dell'affinità tipologica che mostrano con le descrizioni smirneiche, Esposito (1987) 39-43 e Galasso (2003) notano come esse siano debitrici a modelli precedenti. Basti pensare ai frr. 483-484 e 485-486 Skutsch degli Annales di Ennio (in merito cf. anche n. 9), che descrivono le attività residue di due teste amputate, e a un passo dell'opera lucreziana (De rerum natura 3.642-663). In quest'ultimo, come argomento contro l'immortalità dell'anima, sono descritti i movimenti di un serpente tagliato in più parti (per cui vedi anche nota seguente) e, in precedenza, degli arti di guerrieri mutilati dai carri falcati (642-645). Nel brano si nota anche come gli uomini continuino a combattere non accorgendosi subito della mutilazione (645-651), mentre le parti recise si agitano a terra, come un piede che ancora muove le dita (653) o una testa che conserva un volto vivo e gli occhi aperti (654-655). Da notare che la filosofia lucreziana non è l'unica a utilizzare descrizioni spesso macabre per dimostrare le proprie teorie, in particolare sull'esistenza e la natura dell'anima: il procedimento sembra infatti interessare anche la dottrina stoica, come sottolinea Most (1992).

5 L'immagine delle parti tagliate di un serpente che continuano a contorcersi è presente già in Lucr. 3.657-663 (per l'apporto dato dall'opera lucreziana allo sviluppo del gusto del macabro, cf. nota precedente), e in seguito riappare in Ovidio (Metamorphoses 6.555-560), qui, al pari del caso di Q.S. 11.74-76, come termine di paragone di una similitudine (in questo caso però dei tremori di una lingua mozzata). 
ro, che nel suo movimento sembra cercare di partecipare ancora alla mischia, sebbene non sia però più sorretto da forza vitale (77-78). ${ }^{6}$

La descrizione dell'attività residua dell'arto amputato è invece sapientemente variata poco dopo, sebbene il meccanismo seguito sia il medesimo di quello appena citato: la parte staccata dal tronco continua infatti, una volta a terra, a compiere l'ultimo movimento che stava facendo prima di essere recisa. Ai versi 188-200 del medesimo libro, Agenore con un'ascia bipenne colpisce il braccio di un Danao rompendogli l'osso e i tessuti nervosi (190-192). ${ }^{7}$ L'uomo si accascia sul collo del proprio cavallo e muore, ma lascia il proprio braccio stretto alle redini, come se un uomo vivo lo stesse ancora guidando ( $\lambda^{\prime} i \pi \varepsilon\langle v\rangle \delta$ ' $\alpha \rho \alpha \chi \varepsilon i \rho \alpha$

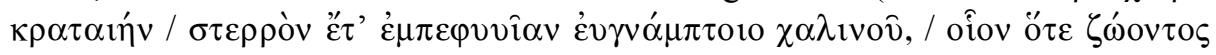
" $\eta v$, "ma lasciò il braccio possente / ancora strettamente aggrappato alle redini ben curvate, / come se appartenesse a un uomo ancora vivo", 194-196). ${ }^{8}$ Il narra-

$6 \quad$ Un caso simile di movimento convulso di una parte del corpo amputata in battaglia si trova anche, come nota Galasso (2003) 164, in un passo dell'Eneide (Verg. Aen. 10.395-396), in cui la mano destra di Laride, amputata dal corpo, muove convulsamente le dita cercando ancora di stringere la spada.

7 In questo caso, al termine impiegato al verso 191, vย̂ิ $\rho \alpha$, si può attribuire il significato unico di tessuto nervoso, piuttosto che in maniera più estensiva, come nell'epica omerica, di parte del corpo fibrosa o di natura tendinea che può quindi indicare anche legamenti e tendini. Nel lessico

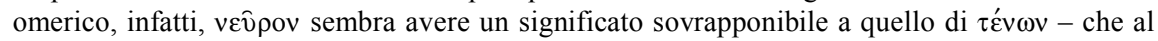
massimo si specializza indicando tendini o legamenti importanti o vistosi, come la doppia proiezione longitudinale dei muscoli del collo (per es. Il. 10.456; 14.466; 16.587; Od. 3.449-450), i tendini del piede (Il. 4.521; 17.290; 22.396-397), del gomito (Il. 20.478-479) o i legamenti dell'anca che collegano la testa del femore all'acetabolo (Il. 5.307) - dal momento che Omero non sembra avere conoscenza delle fibre nervose in quanto tali. Per un'analisi di $\tau \varepsilon ́ v \omega v$ e vยv̂pov nell'epica omerica (e anche per lo studio di îves, in parte accostabile ai primi due termini), cf. Daremberg (1865) 28-29. L'evoluzione dell'uso di questi termini nella letteratura medica, in particolare nei trattati ippocratici De fracturis e De articulis, è messa in luce da Di Benedetto (1986) 228, il quale tra l'uso di vêิpov e di $\tau \varepsilon ́ v \omega v$ rileva "in fieri un processo di specializzazione terminologica, con tenōn riservato al tendine nella sua parte più appariscente" (cf. supra l'abbozzo di diversificazione presente già in Omero). Quinto vive invece in un periodo storico in cui era già presente una consapevolezza almeno parziale dell'esistenza e delle proprietà del sistema nervoso grazie soprattutto alle indagini dei medici ellenistici Erofilo ed Erasistrato. Da notare che lo stesso Apollonio Rodio sembra conoscere la funzione di nervi e cervello nella propagazione del dolore, come si comprende da un passo delle Argonautiche (A.R. 3.761-765) che forse è servito come modello anche a Quinto per il mancamento di Aiace (in merito a questi due brani e alle teorie ellenistiche sul sistema nervoso, cf. infra l'analisi dell'attacco di پ̌ $\tau \eta$ di Aiace).

8 L'immagine ha un antecedente al di fuori del genere epico: una scena simile infatti compare anche nell'opera di Erodoto, più in particolare in Historiae 6.91.2. Qui infatti gli Egineti, mentre cercano di trascinare fuori dalla città settecento popolani per condannarli a morte, se ne fanno sfuggire uno che cerca di rifugiarsi nel tempio di Demetra. Egli, per non essere portato via, si aggrappa alle maniglie del portone del tempio e gli Egineti, non riuscendo ad allontanarlo da lì, gli amputano entrambe le mani, che rimangono appese nel punto in cui si trovavano; per un commento al dettaglio macabro del passo, con bibliografia precedente, cf. Esposito (1987) 5152. Il particolare orrido dell'arto superiore che, una volta amputato, rimane appeso a ciò cui era 
tore però non si limita alla descrizione di questo dettaglio già di per sé macabro, ma con un inciso rivolto al fruitore del testo indugia ancora sull'immagine, sottolineando quanto sia prodigioso quel braccio insanguinato che ancora appeso alle redini semina terrore tra i guerrieri (196-198). Anche in questo caso, l'immagine prosegue con la nota che l'arto potrebbe sembrare ancora guidato da forza vitale (199), e si chiude nell'ultimo verso con l'ulteriore moltiplicazione della sensazione di orrore, mediante la precisazione che, nel braccio attaccato alle redini, il cavallo del Danao continua a portare un resto del proprio padrone ucciso (200).

Un caso a parte, ma sempre riguardante l'attività residua di una parte del corpo di un guerriero dopo la morte, è Q.S. 6.634-638, in cui si descrive l'uccisione di Cleolao da parte di una freccia scoccata da Paride. Qui il narratore, dopo aver sottolineato ben tre volte la morte dell'eroe (635-636), si sofferma sull'attività residua questa volta non di un suo arto, ma di un organo: si tratta del cuore di Cleolao che, attraverso un'immagine di forte impatto, continua a pulsare così

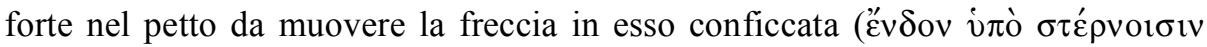

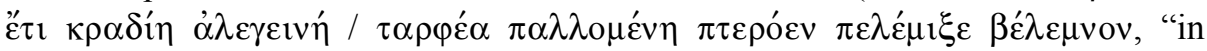
fondo al suo petto il cuore dolorante ancora / pulsando con frequenza faceva vibrare la freccia alata", 637-638). Il passo conserva quindi la descrizione di un'attività residua dopo la morte (sebbene come si è rilevato di natura leggermente diversa rispetto alle precedenti), caricata anche in questo caso di un dettaglio narrativo di forte impatto, ossia il movimento della freccia. Il brano rappresenta un utile esempio anche per comprendere il rapporto che lega, in queste descrizioni, Quinto al modello-codice omerico, dal momento che l'immagine del cuore che pulsando muove un'arma in esso conficcata compare già in $I l$. 13.442444 , che sembra essere servita da ipotesto per il dettaglio smirneico. In questo punto, infatti, Omero descrive come il cuore di Alcatoo, trafitto dalla lancia di Idomeneo, muova l'impugnatura dell'arma con le proprie pulsazioni ( $\delta$ ó $\rho v \delta$ ' $\varepsilon v$

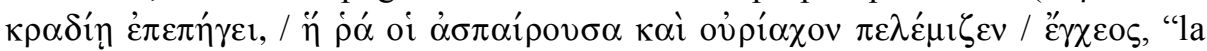

aggrappato prima della recisione si trova anche in Lucano, Bellum civile 3.667-669, passo analizzato da Esposito (1987) 48. Qui è descritto un numero di uomini che, in mare per la perdita della propria nave, cercano di aggrapparsi a un'altra imbarcazione. L'equipaggio di quest' ultima, tuttavia, temendo il peggio, li respinge con violenza tagliando loro le braccia e il narratore si sofferma sul dettaglio macabro degli arti che, una volta recisi, rimangono appesi alla poppa della nave mentre i corpi degli uomini affondano. Una descrizione simile si trova anche poco prima (3.609-617), in cui si allude ancora a una mano che dopo l'amputazione rimane appesa alla nave dei nemici. Un caso simile a quello smirneico appena descritto di arto superiore che, dopo l'amputazione, rimane stretto a ciò cui era aggrappato, ma che si ricollega anche all'esempio precedente dal momento che la mano continua a stringere una spada, si trova in Stazio (Theb. 8.441-444). Il dettaglio della mano che una volta tagliata rimane appesa alle redini, come accade nel passo dei Posthomerica preso in esame, si ritrova invece in un passo dei Punica di Silio Italico (Sil. 4.209-212), per cui, insieme ai due passi precedenti, cf. Most (1992) 397, 399, 406. 
lancia rimase piantata nel cuore, / il quale palpitando faceva vibrare l'impugnatura", 442-444). ${ }^{9}$

A differenza dell'attenzione dedicata alla ricerca del macabro - che come si è rilevato supera spesso la verosimiglianza medica - nella descrizione di alcuni traumi dovuti al combattimento, Quinto mostra un interesse più scientifico, legato maggiormente alle teorie anatomiche e terapeutiche del proprio tempo, quando descrive, in due passi appartenenti al quarto libro, l'attività dei medici del mondo omerico. Anche questi due brevi episodi possono essere inseriti tra le lezioni omeriche, dal momento che sia il contesto in cui si trovano (i giochi funebri in onore di un eroe morto) che i personaggi rappresentati (i medici del campo acheo) sono chiaramente ripresi da Quinto dal modello-genere dell'epica omerica.

9 Una seppur minima verosimiglianza scientifica è abbandonata, a favore della totale intensificazione espressiva creata dall'effetto macabro, nella descrizione smirneica dell'attività residua di teste amputate. In particolare, in Q.S. 11.55-59, un Danao taglia a Piraso la testa, che "rotolando

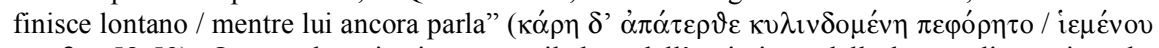
$\varphi \omega \vee \eta ิ \varsigma, 58-59)$. Questa decapitazione, con il dato dell'emissione dalla bocca di suoni anche dopo il decollamento, ne richiama un'altra di impatto macabro leggermente minore, quella di Priamo. In Q.S. 13.241-245, infatti, Neottolemo taglia la testa del re troiano, la quale "emettendo un profondo gemito rotola a terra / ben lontano dalle altre membra grazie a cui un uomo si

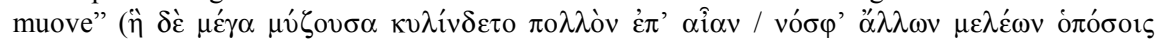
$\dot{\varepsilon}\left\langle\pi \iota<\kappa^{\prime} v v \tau\langle\alpha \downarrow) \dot{\alpha} v \eta \dot{\rho} \rho, 244-245\right)$. In merito al primo passo, il più interessante, bisogna notare come si riallacci a quello che sembra un ipotesto omerico, dal momento che l'immagine ricorre anche in $I l$. 10.455-457, in cui la testa di Dolone, tagliata da Diomede, "si mischiò nella polvere

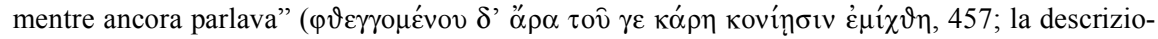
ne compare anche, con lo stesso verso finale, in $O d$. 22.326-329 a proposito della decapitazione di Leode da parte di Odisseo): il brano condivide infatti con quello dei Posthomerica in primo luogo il dettaglio macabro dell'emissione di voce, e inoltre il particolare della recisione dei tendini del collo (Q.S. 11.56-57 $\approx I l .10 .456$ ). Questa descrizione macabra della testa recisa che continua a emettere suoni è presente anche nella letteratura latina, di epoca non solo già matura (cf. per es. Ov. Met. 5.103-106) ma anche arcaica. Due frammenti degli Annales di Ennio (frr. 483-484 e 485-486 Skutsch) ci trasmettono infatti le attività residue di due teste amputate in battaglia, di cui la prima è descritta con la bocca aperta e con gli occhi ancora vivi (oscitat in campis caput a cervice revolsum / semianimesque micant oculi lucemque requirunt, "la testa staccata dal collo sta tra i campi a bocca spalancata / e gli occhi, quasi privi di vita, ancora si muovono in cerca della luce", frr. 483-484 Skutsch). La seconda, invece, quella di un trombettiere, cade a terra, e "la tromba da sola continua a suonare / e si sente un suono rauco nell'aria mentre l'uomo muore" (quomque caput caderet carmen tuba sola peregit / et pereunte viro raucum sonus aere cucurrit, frr. 485-486 Skutsch). Sull'interpretazione di questi passi della letteratura latina - anche in merito alla descrizione della testa amputata nel terzo libro di Lucrezio - e sulla bibliografia a riguardo cf. n. 4. L'immagine si ritrova anche nella produzione di periodo imperiale, una volta persino rovesciata dal momento che è il corpo che continua a muoversi dopo la decapitazione: per quel che riguarda la testa che continua a emettere suoni o parole dopo il decollamento, cf. per es. Seneca, Agamemnon 901-903, mentre nell'opera di Silio Italico (13.246-248) compare il rovesciamento dell'immagine, con il corpo del decapitato che continua a correre per l'impeto che rimane nel tronco; per un'analisi di questi passi alla luce del generale interesse della letteratura di età imperiale per le immagini orride, cf. Most (1992) 406. 
Proprio la brevità e la cursorietà degli episodi (la caduta di Teucro durante la gara di corsa, la cura delle ferite alla fine dello scontro di pugilato), del tutto secondari rispetto alla narrazione, permettono all'autore una maggiore libertà, la possibilità di dipingere due tableaux sull'intervento di medici nell'epoca eroica con una profonda attenzione ai particolari anatomici e terapeutici basata anche su teorie scientifiche come quelle trasmesse dal Corpus Hippocraticum.

Nel primo passo in cui Quinto narra con dovizia di particolari l'attività medica nel campo acheo, descrive con attenzione anatomica la caduta di Teucro durante la corsa, per poi concentrarsi sulle successive medicazioni a opera degli

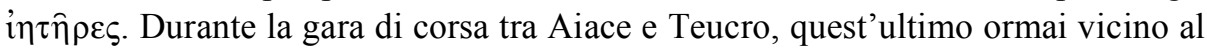
traguardo inciampa su un ramo di tamarisco e torce indietro la parte anteriore del piede sinistro, provocandosi quella che sembra almeno una distorsione (Q.S. 4.203-204). Oltre al dolore provato da Teucro, il narratore descrive l'inizio del gonfiarsi della parte colpita, notando che "si gonfiarono / le vene, tumefatte da

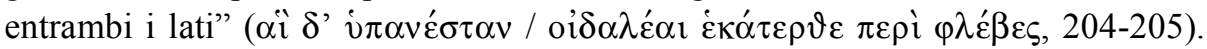
Poco dopo la fine della gara, l'attenzione si concentra sulle cure prestate dai medici al piede di Teucro. Per prima cosa, questi "velocemente [...] / tolgono il

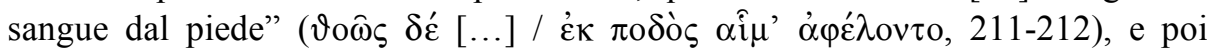
pongono sulle ferite delle medicazioni composte da panni di lana imbevuti di unguenti che placano il dolore (212-214). ${ }^{10}$

Nel secondo episodio, dedicato alla cura delle ferite alla fine delle gare di pugilato, entra in scena uno dei due medici par excellence dell'epica omerica, Podalirio. Mentre però in Omero il figlio di Asclepio, come il fratello Macaone, è descritto, oltre che come medico, come condottiero (nel suo caso degli uomini di Ecalia) che partecipa direttamente ai combattimenti e quando non è impegnato in azioni di guerra si dedica all'arte medica, ${ }^{11}$ nei Posthomerica è ritratto nella sua

10 Il verso 212 presenta un problema di trasmissione, dal momento che l'ultimo termine conservato

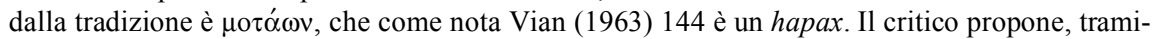

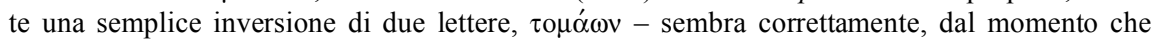

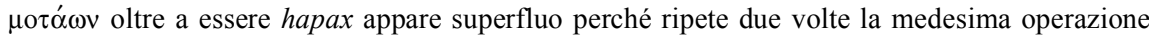
del bendaggio - che potrebbe indicare sia le ferite in generale che le incisioni praticate per far uscire il sangue dalle ecchimosi (sull'importanza della tecnica della flebotomia, uno dei cardini della terapia ippocratica, cf. infra la descrizione del caso successivo; per la cura di casi simili a quello di Teucro nel Corpus Hippocraticum, Vian cita per esempio Ippocrate, De officina medici 22-23 e De fracturis 9-11). Van Krevelen (1964) 178 accetta invece a testo $\mu$ o $\alpha \dot{\alpha} \omega v$ notando come il passo secondo lui sia in stretta correlazione con un punto del trattato ippocratico De vulneribus in capite (14), in cui si riscontra un'alta concentrazione di termini tecnici derivanti dalla stessa radice.

11 Nell' Iliade, infatti, quando Euripilo ferito chiede aiuto a Patroclo, quest'ultimo gli risponde che i due medici principali dell'armata greca non sono disponibili, dal momento che Podalirio è in battaglia mentre Macaone, ferito durante i combattimenti, giace nella sua tenda e ha a propria volta bisogno di un medico (Il. 11.833-836). Sulla doppia funzione di guerrieri e di medici dei due figli di Asclepio, cf. già Daremberg (1865) 6. 
attività di in $\tau$ ń $\rho$. Oltre infatti al suo intervento principale nella trama - ossia la guarigione, grazie all'aiuto del padre, della piaga di Filottete nel libro decimo (cf. infra) - anche qui Podalirio entra in scena per curare delle ferite, in questo caso quelle dei pugili.

La tecnica del medico è descritta in modo breve ma esaustivo: Podalirio cura con rapidità le ferite prima ripulendole tramite un'incisione che fa uscire il

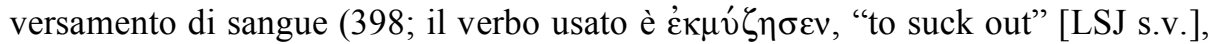
detto letteralmente di sangue, che indica nella sua forma composta e in quella semplice un procedimento terapeutico già praticato in epoca arcaica e poi diventato uno dei cardini della terapia ippocratica) e poi suturandole con le proprie mani esperte (398-399). Infine, il medico pone sopra le ferite "quei medicamenti

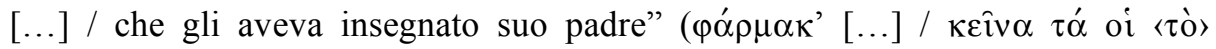

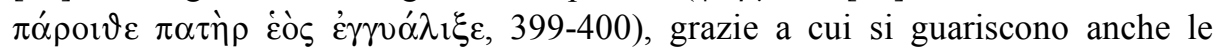
ferite incurabili (401-402).

La tecnica terapeutica di questo passo si trova già accennata in alcuni versi del quarto libro dell'Iliade, in cui il protagonista non è Podalirio, bensì il fratello Macaone, alle prese con una ferita di Menelao. In questi versi, che sembrano fungere da modello-codice per quelli dei Posthomerica sia per il lessico sia per i procedimenti terapeutici, Macaone estraeva dalla ferita la punta della freccia e poi "dopo aver cavato fuori il sangue ci spalmava sopra con arte farmaci lenitivi"

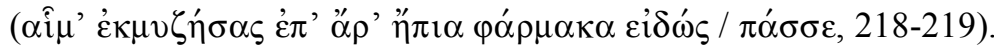

Se si riconsidera quindi la descrizione di Quinto alla luce di questo passo omerico, si nota immediatamente che i dati presenti nei versi dei Posthomerica la tecnica di cura (cf. di nuovo la presenza del verbo $\dot{\varepsilon} \kappa \mu \nu ́ \zeta(\omega)$, l'uso del termine polivalente $\varphi \alpha ́ \alpha \mu \alpha \kappa \alpha-$ sembrano essere in diretta relazione con il modello-codice rappresentato dal passo omerico. ${ }^{12}$

Tra nuove teorie scientifiche e fedeltà al modello della tradizione: la piaga di Filottete e quella di Paride, l'attacco di ő $\tau \eta$ e la follia di Aiace

L'interesse di Quinto per le teorie mediche e la sua volontà di inserirle nei dettagli all'interno della narrazione si scontrano a volte con esigenze di trama legate al mito. Si tratta di casi più complessi rispetto a quelli trattati in precedenza: l'autore ha infatti la possibilità di utilizzare più estesamente le conoscenze mediche della sua epoca storica, ma allo stesso tempo si trova nella necessità di contraddirle

12 Per un'analisi più approfondita di questi temi e della concezione omerica dell'apparizione e della scomparsa della malattia, cf. infra la sezione riguardante la guarigione della piaga di Filottete, in cui queste teorie sono espresse da Quinto più estesamente. 
oppure di ridimensionarle allo scopo di rimanere nei binari della storia narrata dal mito.

Come si può comprendere, la tecnica compositiva di Quinto si scontra con le esigenze della trama soprattutto nella narrazione di episodi, molto famosi e importanti per la storia, che hanno alle spalle le versioni schiaccianti non solo della tradizione omerica, ma più in generale di quella letteraria antica, dalla tragedia all'epica ellenistica, dai cicli epici alla poesia didascalica tarda. In questi casi, le scelte compositive di Quinto non riescono ad amalgamare omogeneamente dettagli medici precisi e scientifici con la trama richiesta dal mito. L'autore quindi inserisce nella narrazione le proprie innovative descrizioni anatomiche o fisiologiche per poi tornare bruscamente, quando il racconto lo richiede, alla narrazione e alle concezioni mediche tradizionali.

Due casi in particolare risultano paradigmatici per mettere meglio in luce questo meccanismo compositivo: la descrizione della piaga al piede e della guarigione miracolosa di Filottete nel nono libro, e quella della ferita mortale di Paride e degli oracoli sulla sua possibile guarigione nel libro successivo. Proprio la narrazione della piaga di Filottete (e anche di quella di Paride, che gli fa eco in quanto originata dallo stesso veleno) permette di comprendere la continua tensione di Quinto tra adesione a temi tradizionali e spinta verso descrizioni più scientifiche, quasi macabre.

La parte centrale del nono libro dei Posthomerica è dedicata all'arrivo a Troia di Filottete, abbandonato precedentemente a Lemno a causa della sua ferita al piede dovuta al morso di un serpente velenoso. ${ }^{13}$ A capo dell'ambasciata sull'isola sono mandati - secondo la versione più seguita del mito - Odisseo e Diomede, che quando arrivano presso la caverna di Filottete sono colpiti da una serie di elementi, in particolare l'ambiente in cui l'eroe è costretto a vivere e la sua persona. I primi diciassette versi sono dedicati a una serie di tableaux che descrivono la caverna e il vestiario dell'eroe. Il dato che colpisce è che Filottete è circondato da uccelli, sua unica preda, che non rappresentano però solo il cibo, ma tutto il mondo del malato. Le piume infatti costituiscono il giaciglio su cui dorme, ma anche, cucite assieme, il vestito che indossa (Q.S. 9.357-360); ${ }^{14}$ a questo si aggiunga che esse servono anche come cibo e come bendaggio, o alme-

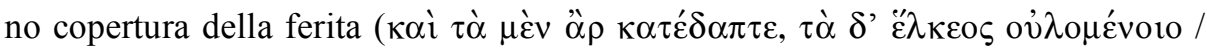

13 Per uno sguardo d'insieme sulla diversa identificazione del serpente che morde Filottete non solo nella letteratura, ma anche nei commenti dell'antichità, cf. Vian (1966) 177-178.

14 Da notare che l'uso massivo delle piume in ogni aspetto della vita di Filottete sembra un'innovazione di Quinto dal momento che, per esempio, nel Filottete di Sofocle la descrizione della caverna dell'eroe non le comprende, ma allude a un giaciglio di foglie (Soph. Ph. 33) e a stracci stesi (38-39). I volatili, in particolare le colombe, sono menzionati solo come cibo (287289; e anche 710-712; 955-956; 1092-1094; 1146-1151), mentre per tamponare la piaga si parla solo di foglie (696-699). 


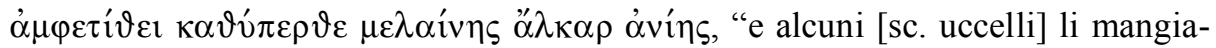
va, altri / li poneva sopra la maledetta piaga per placare i suoi neri dolori”, 362363; in merito alla natura della ferita, pare molto importante e tornerà anche in seguito l'indicazione del suo colore nero - qui resa tramite enallage con il dolore provato da Filottete - che nasconde una caratterizzazione della piaga innovativa e fondamentale dal punto di vista medico). ${ }^{15}$

Dopo la descrizione dei suoi capelli simili a quelli di una bestia selvaggia presa alla zampa in una trappola, la quale pur di liberarsi è disposta a staccarsela con i propri denti (immagine ardita che riprende il sottofondo intertestuale del Filottete di Sofocle, in cui più volte il protagonista, in preda ai dolori, supplica Neottolemo di amputargli il piede), il narratore passa a descrivere, attraverso il punto di vista di Odisseo e Diomede, la situazione di salute di Filottete tramite una dettagliata anamnesi, che ricorda quelle dei trattati medici antichi, di ogni sintomo e ogni caratteristica corporea. Il fisico dell'eroe è ridotto pelle e ossa, emana un pessimo odore, gli occhi sono scavati nelle orbite e il suo dolore è continuo, dal momento che "la nera piaga gli è giunta fino all'osso" (oi $\mu \varepsilon ́ \lambda \alpha \nu$

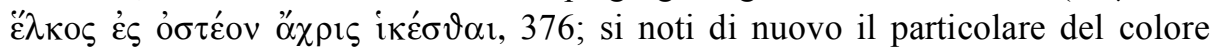
della ferita). Il dato del dolore che rode il corpo (esattamente come la piaga) è ripetuto anche al verso successivo ed è ribadito da una similitudine, in cui la ferita che si estende è paragonata all'azione corrosiva del sale, dei venti e delle onde su di uno scoglio marino affiorante. Segue la prima esplicitazione (385-386, la seconda si trova poco dopo, 394-395) dell'incurabilità della ferita, causata da un serpente al cui veleno non c'è scampo. Chiude questa precisa e macabra descrizione del corpo di Filottete la menzione dell' "umore" ('i $\chi \omega \rho$, ossia una sierosità nociva ritenuta dagli antichi causa della necrosi dei tessuti e la cui flussione da piaghe era presagio di esito infausto, 390$)^{16}$ che continua a gocciolare fuori dalla piaga (389-390), elemento, come nota correttamente Vian, che appartiene alla rappresentazione tradizionale della piaga dell'eroe. ${ }^{17}$

Come si può notare dalla sintesi di questa sequenza, Quinto descrive in maniera molto precisa, come al solito a tratti scadente nel macabro, la piaga di Filottete: la ferita risulta infetta e purulenta (come si nota dall'indicazione del pus

15 In Omero e in Sofocle, infatti, non si allude mai al colore nero della ferita di Filottete, ma al

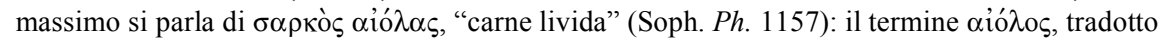
da LSJ s.v. a livello di colorazione genericamente con "changeful of hue, sheeny", nello specifico caso del Filottete è indicato dal lessico con valore di "discoloured", forse in considerazione del colore assunto dai tessuti affetti da necrosi. In merito più in particolare alle concezioni mediche presenti nella tragedia greca, dall'anatomia e fisiologia alla patologia fino ai metodi terapeutici, cf. Dumortier ( $\left.{ }^{2} 1975\right)$, sui drammi eschilei, e più in generale Guardasole (2000).

16 In merito al valore attribuito all' 'x $\chi \omega$ in passi tragici e non solo, cf. Guardasole (2000) 130-143.

17 Basti pensare, con Vian (1966) 221, a Soph. Ph. 7; 39; 696-697; 783-784 e più in generale, sulle ferite provocate da serpenti, a Nicandro, Theriaca 235 (in cui si trova il termine i $\chi \dot{\omega} \rho$ ); 362-363. 
di cui è coperta, 377) e ormai sembra essere in necrosi, con la formazione di una cancrena in continua espansione. Questo elemento è ben messo in luce dal narratore, che in primo luogo descrive la ferita come nera (363 tramite enallage e 376), e sceglie inoltre molto spesso verbi o intere immagini che indicano il procedere della cancrena che intacca la parte sana: si pensi per esempio ai "dolori che

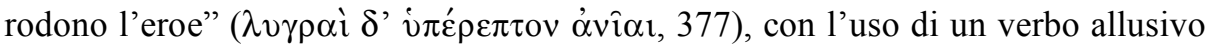
in questo senso, al dettaglio della ferita che si espande a causa del veleno (383384) e alla similitudine dello scoglio attaccato da fattori di erosione (378-382). ${ }^{18}$

Anche in questo caso, la descrizione a opera di Quinto di un aspetto del mito riguardante in qualche modo la medicina risulta attenta al dettaglio, non solo dell'affezione in senso stretto ma anche delle condizioni generali della persona. L'infezione e l'estensione necrotica della ferita di Filottete sono infatti descritte nei minimi dettagli, fino, come si è rilevato anche per altri brani, a toccare il macabro. Molta attenzione inoltre è dedicata, oltre al degrado in cui vive l'eroe, alle sue condizioni generali: peso, colorito, occhi, odore, condizione dei capelli e altri dettagli fisici.

Dopo questa descrizione clinicamente molto precisa - soprattutto se la si considera inserita in un contesto epico che non richiedeva tale attenzione - e in

18 Non a caso, sebbene la piaga che affligge Filottete non sia ancora descritta con precisione

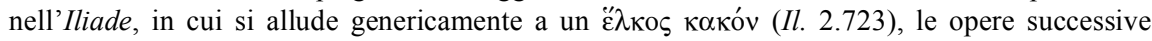
riportano espressioni fortemente caratterizzate a favore di una ferita necrotica. Tutte infatti alludono etimologicamente all'azione di divorare il piede, alcune chiamando ciò che affligge l'eroe con il termine più preciso $\varphi \alpha \gamma \varepsilon ́ \delta \alpha \imath v \alpha$ - come Eschilo nel proprio Filottete (fr. 253 Radt,

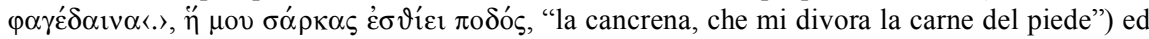

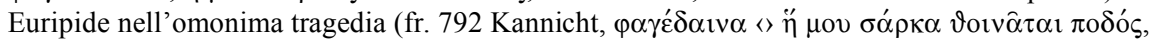
"la cancrena che mi divora in continuazione la carne del piede") - altre usando un aggettivo derivante comunque dalla radice $\varphi \alpha \gamma-$, come accade nel Filottete sofocleo (Soph. Ph. 313,

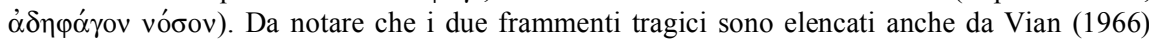
195, che, senza menzionare il passo del Filottete citato sopra, aggiunge altri due casi leggermente meno accostabili ai primi ma in cui compare comunque l'immagine del divorare o del

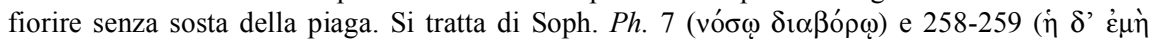

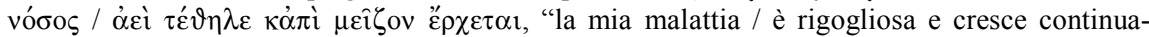
mente"). In merito a $\varphi \alpha \gamma \varepsilon ́ \delta \alpha ı \alpha$, cf. tra gli altri Andorlini / Marcone (2004) 42, i quali inseriscono il termine all'interno del processo storico di formazione di una terminologia medica precisa. Da notare anche l'analisi di questi passi frammentari a opera di Guardasole (2000) 240244, che individua nella piaga di Filottete un esempio di quella che ancora oggi è chiamata

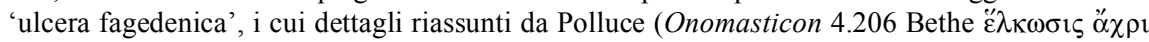

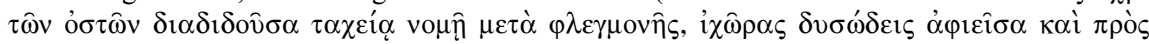

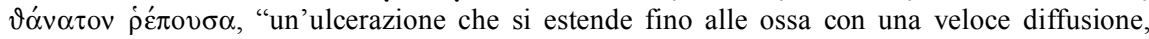
unita a flemmone sieroso, di cattivo odore e che porta alla morte") ricordano da vicino quelli della ferita di Filottete nei Posthomerica. La studiosa riporta inoltre un passo di Ippocrate (De ulceribus 10.5) in cui nella descrizione dell'ulcera fagedenica si sottolinea anche il colore nero livido che assumono i tessuti della zona circostante la ferita, come accade nella descrizione smirneica. Riguardo invece all'immagine dello scoglio eroso poco alla volta dai fenomeni atmosferici, cf. Vian (1954) 46 e 241. 
linea con le conoscenze mediche del tempo, l'autore si trova a questo punto davanti due elementi tra loro inconciliabili ma entrambi evidenti: l'impossibilità di guarigione della piaga con i mezzi della scienza medica antica e il completo ritorno in salute di Filottete, la cui presenza è necessaria per la presa di Troia. Questa tensione tra due poli opposti è risolta dall'autore tramite uno scarto: dopo aver descritto la piaga di Filottete secondo le teorie mediche più avanzate del suo tempo e averne prognosticato l'incurabilità e l'inevitabile degenerazione, Quinto si riallaccia infatti al racconto mitico ritornando alla concezione della malattia e ai metodi di terapia descritti dalla tradizione omerica e tragica.

Dall'analisi del testo le maglie di questo meccanismo risultano evidenti: durante la precisa descrizione della situazione in un certo senso 'clinica' di Filottete, infatti, il narratore ribadisce più volte l'incurabilità e l'inevitabile degenerazione della ferita dell'eroe. Oltre a varie volte in cui descrive la piaga come genericamente terribile o dolorosa, quando si sofferma sulla sua origine, ossia il morso velenoso del serpente, Quinto si premura di ricordare come le ferite che l'animale infligge siano ritenute incurabili (385-386), e poco dopo aggiunge di nuovo che per questo l'eroe è preda di un dolore non curabile (388), esattamente come la sua ferita. L'affermazione è ripetuta poco dopo, quando il narratore, soffermando la propria attenzione sulle frecce di Filottete intrise nel veleno del serpente, nota ancora come per la sostanza non esista cura (394-395) e in seguito, creando quasi un tableau di Odisseo e Diomede in visita al capezzale di un malato, di nuovo aggiunge il particolare, oltre che del terribile dolore, della letalità della ferita (408).

Partito da questo elemento, Quinto opera uno scarto riallacciandosi al racconto della tradizione e, per spiegare la completa guarigione richiesta dal mito, accoglie le terapie e le concezioni mediche presenti nei propri modelli letterari. Dopo averlo infatti lavato, Odisseo e Diomede portano Filottete con loro a Troia e a questo punto avviene la guarigione, del tutto miracolosa, a opera del figlio di Asclepio Podalirio. ${ }^{19}$ È infatti lui, indicato come uguale agli dei celesti (463), che cura Filottete con una tecnica del tutto simile a quella presentata spesso nell'epica omerica e che invoca il proprio padre in soccorso della guarigione (464-465). A questo punto l'eroe torna in salute, guarigione indicata di nuovo da Quinto come chiaramente miracolosa, ossia dovuta solamente alla volontà divina. Grazie proprio agli dei, infatti, il dolore e la prostrazione di Filottete spariscono (468469), e in seguito il narratore commenta come gli Argivi, che attribuivano la

19 Da notare che la guarigione 'mitica' dovuta all'intervento divino (come avviene nei Posthomerica) è presente a un livello ancora più profondo anche in Sofocle: nel Filottete infatti Eracle predice che la guarigione dell'eroe avverrà grazie non ai figli di Asclepio, come aveva accennato Neottolemo (Soph. Ph. 1333-1334), ma al dio in persona (1437-1438). 
guarigione dell'eroe alla volontà divina, avessero ragione, dal momento che era stata Atena in persona a ridare vigore e bellezza al corpo di Filottete (481-485).

In linea con la concezione omerica della malattia e della guarigione, entrambe attribuite alla volontà divina, Quinto si riallaccia alla tradizione epica precedente anche nei particolari del procedimento di Podalirio per curare Filottete, dal momento che, allontanandosi da una qualsiasi terapia medica reale, l'autore ricalca non solo i gesti, ma anche il lessico estremamente vago delle cure mediche del mondo omerico. Podalirio, infatti, oltre a invocare il nome del proprio padre, agisce solamente "ponendo sopra / la ferita molti balsami efficaci" ( $\varepsilon \hat{v} \mu \grave{\varepsilon} v$

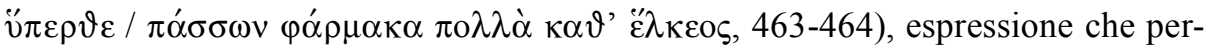
mette di comprendere come, anche dal punto di vista terminologico, l'autore sacrifichi la precisione scientifica, dal momento che utilizza una parola omerica per eccellenza, polivalente e allo stesso tempo estremamente vaga come $\varphi \alpha ́ \rho \mu \alpha \kappa \alpha .{ }^{20}$

Lo stesso scarto tra scienza medica e modelli tradizionali è operato da Quinto nella descrizione della ferita di Paride, momento speculare, sebbene ridotto, rispetto alla narrazione della piaga di Filottete. In questo caso la situazione iniziale è la medesima - il figlio di Priamo è ferito da una freccia cosparsa con il veleno del serpente che aveva morso Filottete - ma l'esito è capovolto, dal momento che la ferita mortale di Paride, che può essere curata solo per intervento divino attraverso le mani della moglie Enone, a causa del rifiuto di quest'ultima porterà l'eroe alla morte. Si ripresenta quindi, anche se su scala ridotta, la medesima tecnica compositiva del caso precedente, ossia la tensione tra scienza medica antica e racconto mitico, e, a causa delle esigenze di scorrimento della trama, il brusco ritorno dell'autore alle concezioni mediche e terapeutiche dell'epica omerica.

Durante il combattimento del decimo libro, Paride è ferito da una freccia scoccata da Filottete ed è costretto a ritirarsi dalla battaglia (Q.S. 10.239-245). In seguito, il narratore sottolinea come l'impegno dei medici non dia nessun sollievo alla ferita dell'eroe ma risulti anzi del tutto improduttivo, a causa della profezia secondo cui Paride sarebbe sfuggito alla morte solamente grazie alle cure di Enone (260-263). Anche in questo caso, quindi, la scienza medica da sola non può nulla, dal momento che Paride si salverà solo per volere degli dei se seguirà le prescrizioni dell'oracolo.

Di nuovo, la piaga mortale dell'eroe è descritta secondo gli stessi patterns narrativi e gli stessi dettagli usati in precedenza per quella di Filottete. La ferita di Paride è nera sulla superficie e in profondità, indice anche in questo caso di quella

20 Il termine infatti nell'epica omerica indica tutto il range delle sostanze farmacologiche, dai veleni alle droghe (stimolanti o tossiche) fino all'ampia gamma di rimedi benefici. Per un utile sguardo d'insieme sull'uso del termine pó $\rho \mu \alpha \kappa o v$ e sui metodi di cura nella medicina omerica, cf. Andorlini / Marcone (2004) 2-6. 


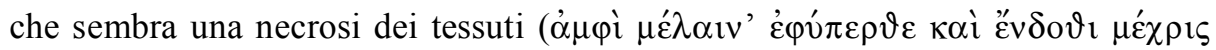

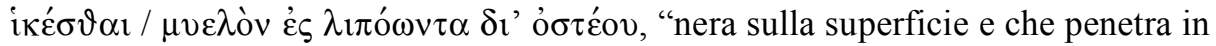
profondità / attraverso l'osso fino al grasso del midollo", 273-274). Tra la cancrena di Filottete e quella di Paride c'è tuttavia una differenza sostanziale, che rende la seconda decisamente più maligna della prima: la necrosi di Paride infatti non si trova in una zona periferica del corpo ma nel ventre, che risulta ormai completa-

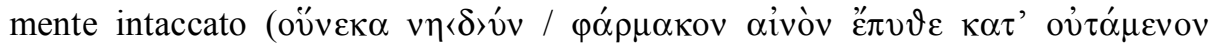
$\chi \rho o ́ \alpha$ $\varphi \omega \tau$ ó $\varsigma$, "poiché / il terribile veleno ha mandato in putrefazione il ventre dove la pelle è stata ferita dalla freccia", 274-275). A questo si aggiunge la menzione dei dolori che attanagliano il petto di Paride esattamente - attraverso una similitudine sempre di ambito medico - come la sete prende un uomo malato, probabilmente affetto da una qualche febbre, facendogli bruciare il petto, infiammare la bile e seccare le labbra (276-281).

Nonostante quindi la ferita di Paride si presenti come maligna e anzi più difficile da curare di quella di Filottete, Quinto tuttavia, operando uno scarto rispetto alle concezioni scientifiche della medicina antica, si riallaccia alla tradizione omerica notando più volte come anche questa piaga potrebbe essere guarita per volere degli dei e adottando il lessico e le concezioni mediche del suo modello epico. Anche qui il narratore sottolinea come le cure mediche non riescano a guarire la ferita di Paride, dal momento che il suo destino vuole che lui possa evitare la morte solo grazie alla moglie Enone (260-263; il concetto è ribadito nel discorso di Paride a quest'ultima, 291-294). Si tratta quindi, come nell'epica omerica, di un caso di guarigione dovuta alla volontà divina: questo dato in seguito è ribadito ulteriormente dal narratore, il quale ricorda che Paride aveva già saputo di tutto questo tramite una profezia (263) e che, mentre subito dopo il ferimento l'eroe cerca di convincersi che il volo funesto degli uccelli intorno a lui non significhi nulla, in realtà esso sta davvero a indicare la sua prossima morte (265-269).

Un altro elemento a favore del legame di questo brano con le concezioni mediche dell'epica omerica è l'attenzione che Quinto dedica al personaggio nelle cui mani risiederebbe, per volere divino, la guarigione di Paride, ossia Enone. Nell'epica omerica infatti molta importanza è data a figure femminili che conoscono l'arte dei $\varphi \alpha ́ \rho \mu \alpha \kappa \alpha$ e possono quindi curare tramite l'intervento divino: ${ }^{21}$ da Agamede figlia di Augia (Il. 11.738-741), a Polidamna, sposa di Tone, che si dice aver fornito a Elena medicamenti di origine egiziana (Od. 4.227-230), fino tralasciando Circe, che è caratterizzata piuttosto come una maga vera e propria - a

21 Alle figure femminili con poteri di 'guaritrici', anche tramite droghe sconosciute o di cui non si fa diretta menzione, accenna già Daremberg (1865) 9; in merito a questo aspetto dell'epica omerica, cf. anche Andorlini / Marcone (2004) 12. 
Elena stessa, che nell'Odissea prepara un medicamento miracoloso per Telemaco $(4.219-234){ }^{22}$

Anche a livello lessicale in questo brano, come nella descrizione della ferita di Filottete, Quinto adotta una terminologia vaga del tutto simile a quella dei suoi modelli-genere, per esempio l'uso di póp $\mu \alpha \kappa o v$ nel suo valore più esteso. Il termine occorre, sempre come possibile terapia coadiuvata dall'intervento divino, nel discorso con cui Paride cerca di convincere Enone a salvarlo. L'eroe, infatti, supplica la donna di guarirlo tramite lo stesso processo con cui Podalirio salva Filottete, ossia "applicando balsami salvifici sulla ferita mortale" ( $\varphi \alpha ́ \rho \mu \alpha \kappa$ '

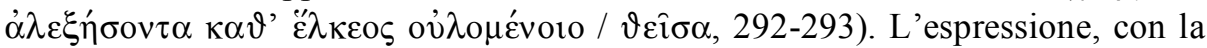
presenza di $\varphi \alpha ́ \rho \mu \alpha \kappa o v$ e la sua generale vaghezza, riprende specularmente quella già citata del caso di Filottete e pare anche in questo caso riallacciarsi alla tradizione omerica che Quinto usa come modello-genere.

Più complesso è il caso delle due scene riguardanti Aiace nel quinto libro, ossia il primo attacco di ö $\tau \eta$ dopo che le armi di Achille sono assegnate a Odisseo e la successiva follia che porterà alla strage degli armenti e al suicidio dell'eroe. Se si considera più da vicino la struttura della narrazione del libro, si nota che, in seguito alla scelta da parte dei prigionieri troiani di donare le armi di Achille a Odisseo, il narratore focalizza l'attenzione sulla reazione devastante di Aiace e in seguito sul suo attacco di follia. Mentre Odisseo trabocca di gioia, infatti, l'esercito irrompe in un grido di dolore; Aiace rimane invece raggelato dalla notizia, e ó $\tau \eta$ - nel suo significato omerico di "mental blindness", come notano James / Lee (2000) 107 - piomba su di lui (Q.S. 5.322-323). ${ }^{23}$ In seguito l'eroe è portato dai suoi nella tenda, e solo dopo, durante la notte, Atena fa crescere in lui la follia vera e propria, quella che lo spinge a fare stragi delle greggi immaginando che si tratti dei capi achei, e che poi, una volta svanita, lo porta alla tragica constatazione dell'errore e al suicidio.

22 Il passo dell'Odissea (limitandosi a 4.227-230) è citato anche da Erodoto (2.116.4) a dimostrazione che Omero conoscesse le peregrinazioni in Egitto di Paride ed Elena, deviati in questo luogo da venti contrari durante il viaggio di ritorno verso Troia. Come notano correttamente Andorlini / Marcone (2004) 12, l'attribuzione a figure femminili di competenze nell'uso di droghe curative non riguarda solo l'epica omerica, ma anche più in generale la tradizione letteraria antica.

23 Vian (1966) 30 non rileva il problema e traduce il passo con "d'un seul coup, le triste Malheur fond sur lui". Tuttavia, come nota García (1986) 111 sulla scia delle teorie di Dodds (1951), questo è il passo dei Posthomerica in cui il termine sembra assumere in modo più evidente il significato di "bewilderment, infatuation, caused by blindness or delusion" (LSJ s.v.) - ossia l'annebbiamento temporaneo della coscienza normale causato da un'entità sovrannaturale presente soprattutto nell'Iliade e in declino già nell'Odissea. Nelle altre occorrenze dei Posthomerica, secondo il critico, il termine assume invece il valore più recente di "avversità, disgrazia, sfortuna o destino avverso" (cf. per es. Q.S. 3.660; 4.201; 5.164; 5,469; 11.470; 12.487; a parte è il caso di 1.753, in cui compare la personificazione divina di ö $\tau \eta)$; per il significato del termine cf. anche n. 30 . 
Nella prima parte della narrazione, quella del mancamento dell'eroe, i sintomi che Aiace accusa sono descritti con la solita precisione, seguendo come si vedrà le opinioni mediche più avanzate. Le prime reazioni fisiologiche interessano il sangue e la bile nera: il primo ribolle nelle vene dell'eroe, ${ }^{24}$ mentre "la bile nera

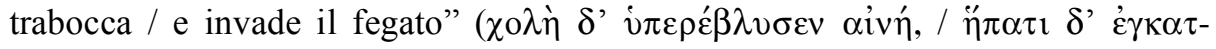
$\varepsilon ́ \mu 1 \kappa \tau$, 324-325). A questo punto Quinto passa dalle reazioni fisiologiche a quelle nervose, soffermandosi sul lancinante dolore che dal cuore, passando attraverso la base del cervello, giunge alle meningi obnubilando completamente la

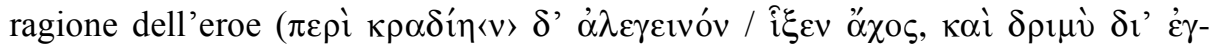

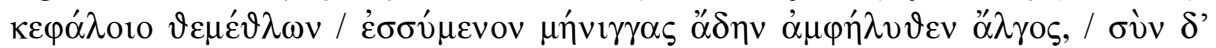

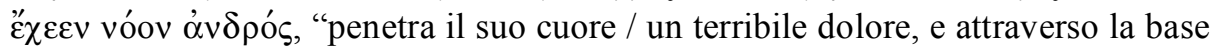
del cervello / il male pungente si irradia nelle meningi / e obnubila completamente la sua ragione", 325-328). Durante questa reazione fisiologica e nervosa, Aiace rimane completamente immobile, con lo sguardo fisso a terra (328-329), finché i suoi compagni non lo conducono alle navi tentando di confortarlo (329331).

Questa descrizione a opera di Quinto del malore di Aiace, come notano James / Lee (2000) 107-108, si può ripartire in due parti distinte - una riguardante le reazioni fisiologiche all'interno del corpo dell'eroe, l'altra invece l'irradiarsi del dolore dal cuore al cervello - che seguono da vicino le teorie della scienza medica del tempo. Le reazioni fisiologiche che avvengono nel corpo dell'eroe, il sangue che ribolle e soprattutto il travaso di bile nera che invade il fegato, riflettono infatti la teoria medica ippocratica dei quattro umori (sangue, flegma, bile gialla e bile nera). Non solo, come rilevano James / Lee (2000), le proporzioni di questi quattro liquidi determinano il carattere di una persona, ma soprattutto elemento messo in luce da van Krevelen (1964) 179-182 - l'aumento esorbitante di uno di essi, nel nostro caso la bile nera, provoca nell'organismo una $\delta v \sigma-$ $\kappa \rho \alpha \sigma i ́ \alpha$ che porta inevitabilmente alla malattia. Come nota il critico, infatti, la concezione ippocratica e in seguito galenica della malattia deve moltissimo alla norma già filosofica, seguita tra gli altri da Empedocle e dai Pitagorici, secondo cui la rottura dell'isonomia (ossia dell'equilibrio all'interno del corpo umano) causa l'insorgere della patologia. È proprio quindi il travaso della bile che provoca un forte scompenso all'interno del corpo di Aiace, e Quinto lo inserisce nella

24 Il verbo usato per indicare questo bollore, $\zeta^{\prime} \omega$ (324) comparirà anche poco dopo, durante l'imperversare di Aiace in preda alla follia causata da Atena, nella similitudine che paragona il bollire del cuore (e in seguito dell'animo) dell'eroe a quello di un calderone posto sopra il fuoco (379-385). Per le diverse ipotesi riguardo al rapporto fra questo passaggio e Verg. Aen. 7.461466 (la furia di Turno paragonata al traboccamento di un calderone), anche in relazione al primo comparire della similitudine della pentola bollente, Il. 21.361-367 (applicato però non all'ira ma alle Rive dello Xanto che bruciano) e alla teoria lucreziana che la propensione all'ira sia dovuta all'eccesso di calore nell'animo (Lucr. 3.288-289 e 294-298), cf. Gärtner (2005) 96-100. 
sua precisa descrizione delle reazioni fisiologiche nel corpo dell'eroe. Da non dimenticare, oltretutto, che in particolare il travaso di bile nel fegato, proprio la reazione devastante che avviene nel corpo di Aiace, era ritenuto dalla medicina antica un evento gravissimo di esito anche mortale. ${ }^{25}$

Sempre calcando sulla gravità della situazione in cui versa Aiace, Quinto aggiunge alla prima parte una seconda che descrive le sensazioni dolorose che si irradiano dal cuore dell'eroe fino ad arrivare al cervello. Anche questa descrizione delle vie che lo stimolo doloroso percorre fino al cervello segue da vicino le teorie mediche dell'epoca: basti pensare per esempio all'attenzione attribuita ai lobi occipitali dai primi investigatori del sistema nervoso, Erofilo ed Erasistato. ${ }^{26}$

Riguardo a questa sezione del mancamento di Aiace, bisogna inoltre rilevare che Quinto si confronta non solo con la scienza medica antica, ma anche con un sottofondo di interesse per le teorie mediche (soprattutto in seguito alle scoperte di età ellenistica) a cui si riallaccia anche la descrizione a opera di Apollonio Rodio nelle Argonautiche (A.R. 3.761-765) del dolore provocato a Medea dagli strali dell'amore. ${ }^{27}$ Anche in questo caso, il dolore che prova la protagonista è descritto mentre si propaga sempre più a fondo, attraverso quello che sembra essere un primo abbozzo di sistema nervoso, ${ }^{28}$ fino al cervello, dove è detto penetrare più ferocemente. ${ }^{29}$

In questo passo, Quinto sembra mostrare in conclusione un'approfondita conoscenza delle teorie mediche del tempo, oltre all'usuale interesse per le descrizioni anatomiche e fisiologiche particolareggiate. Riguardo invece alla tratta-

25 In merito, cf. per esempio il passo del Corpus Hippocraticum (Epistulae 23, sulla bile verde) citato da van Krevelen (1964) 182; le conseguenze sull'organismo della fuoriuscita di bile si ritrovano anche in Ippocrate, De internis affectionibus $48=$ De diebus iudicatoriis 3 .

26 In merito soprattutto alla prima intuizione dell'esistenza di un sistema nervoso ad opera di Erofilo, cf. von Staden (1989), e in parte anche Fraser (1972) I 352; II 512-513.

27 Il passo delle Argonautiche è menzionato cursoriamente, senza un approfondimento del suo rapporto con i Posthomerica, da Vian (1966) 15-16 e James/Lee (2000) 107-108. Per un'analisi del brano cf. tra gli altri Hunter (1989) 179-180 (con bibliografia precedente).

28 Come infatti rileva giustamente Hunter (1989) 180, $\alpha \rho \alpha i \grave{\alpha} \varsigma$ îvos (A.R. 3.762-763) "are most

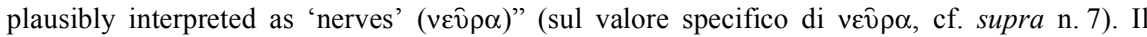
significato di "nervi" che îvaৎ assume nel passo di Apollonio, in linea con le scoperte del periodo ellenistico sul sistema nervoso, è ben argomentato prima di Hunter da Solmsen (1961) 195197, che nota anche che "this is not the only instance in which an Alexandrian poet shows himself abreast with contemporary developments in the sphere of scientific research. The Hellenistic poeta doctus knows how to incorporate the modern and the up-to-date in his supposedly tradition-bound form" (196).

29 In epoca ellenistica, Apollonio Rodio non sembra comunque l'unico autore interessato alle scoperte mediche recenti: anche Callimaco, infatti, pare inserire in alcuni punti delle proprie opere le teorie mediche di Erofilo, come dimostrano Oppermann (1925), in merito alla struttura dell'occhio, e Most (1981), il quale all'interno dell'Inno ad Apollo individua, nella posizione che Leto assume per partorire Apollo, un richiamo alle recenti teorie erofilee di ambito ostetrico. 
zione della causa più generale della follia dell'eroe, su cui la tradizione precedente risulta fondamentale e schiacciante, l'autore sceglie di motivarla ricorrendo ad 'argomentazioni' del tutto tradizionali, addossandone la responsabilità a un intervento sovrannaturale. In primo luogo infatti il mancamento dell'eroe è, nonostante la singola precisione dei dettagli medici con cui è descritto, indicato con il termine $\alpha^{\prime} \tau \eta$, che solo in questo passo smirneico si avvicina al suo significato più arcaico di obnubilamento, causato di solito da una volontà divina. ${ }^{30}$ Inoltre, dato fondamentale, nella seconda scena, il vero e proprio attacco di pazzia di Aiace a causa del quale l'eroe farà strage degli armenti, Quinto si ricollega direttamente alla tradizione precedente - in particolar modo quella tragica, che vede spesso negli avvenimenti una doppia responsabilità, umana e sovrannaturale - motivando la follia di Aiace con una volontà divina, più in particolare il desiderio di vendetta di Atena.

Il narratore infatti, come i suoi ipotesti (soprattutto l'Aiace sofocleo), esplicita la causa della follia dell'eroe attribuendola ad Atena che obnubila volutamente il suo intelletto. ${ }^{31}$ È la dea che fa scendere su Aiace la pazzia (Q.S. 5.360), come accade nella tragedia di Sofocle, ${ }^{32}$ ed è sempre lei - elemento come notano Kakridis (1962) 63 e James/Lee (2000) 128 non precisato nella tradizione precedente - che dopo la strage delle greggi lo libera dalla follia (451-452). Dopo l'allontanamento della personificazione della pazzia, il dato della causa divina della follia di Aiace viene di nuovo ribadito, questa volta attraverso il punto di vista del protagonista. Egli stesso, infatti, dopo aver pensato di essere stato ingannato dagli dei (457-458), ribadisce che sono stati chiaramente loro a oscurare la

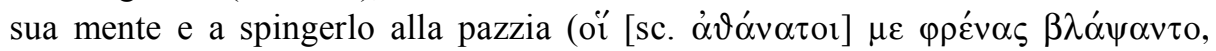

30 García (1986), infatti, riprendendo le concezioni di Dodds (1951), nota come, sebbene in questo preciso passo di Quinto non se ne faccia diretta menzione (cf. però la successiva follia di Aiace, causata da Atena), il termine nel suo significato più arcaico presente nell'Iliade implichi un intervento divino, o quanto meno di un'entità astratta, che infonda appunto ö $\tau \eta$ nell'eroe. In merito al significato del termine, in questo passo e più in generale, cf. anche n. 23.

31 Sulla scarsità di termini medici tecnici nella descrizione della follia di Aiace a opera di Sofocle, che rivolge il proprio interesse sul lato umano della patologia piuttosto che su quello medico e si

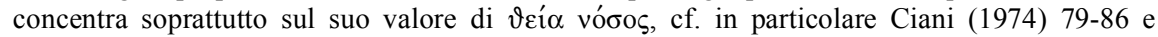
Guardasole (2000) 176-181, la quale, riguardo al tecnoletto medico, nota come quello presente nell'Aiace sia per lo più mutuato da precedenti eschilei (180).

32 Da notare, per esempio, nel dialogo tra Atena e Odisseo l'affermazione della dea (Soph. Aj. 45), che sembra essere stata presa a modello anche da Quinto per il suo narratore, che Aiace sarebbe riuscito nei propri propositi omicidi sui capi argivi se non fosse intervenuta Atena in persona. Poco dopo, infatti, come nel racconto dei Posthomerica, si aggiunge come mai l'eroe non sia riuscito nel suo intento: la dea gli ha gettato sugli occhi immagini ingannevoli e lo ha incitato mentre era in preda alla follia (in particolare, 51-52 e 59-60). Il fatto che il motivo che ha spinto Aiace a fare strage delle mandrie sia assolutamente allotrio rispetto alla sua persona, in quanto causato da una volontà divina, viene ribadito anche nel primo stasimo del dramma, in cui il coro nota come la follia di Aiace non sia probabilmente dovuta a un impulso della sua mente quanto piuttosto al capriccio divino (182-187). 


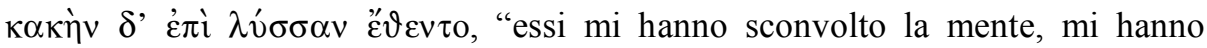
gettato nella tremenda follia", 466).

In questo caso ben si nota come Quinto, nella narrazione dei sintomi specifici dell'attacco di $\alpha$ $\alpha \eta$ di Aiace, momento non fondamentale per lo scorrimento della trama, si senta più libero nell'uso dei mezzi narrativi e quindi inserisca descrizioni anatomiche e fisiologiche dettagliate. Diverso è invece il comportamento dell' autore, più legato alla concezione tradizionale della responsabilità degli avvenimenti che colpiscono gli umani, nella seconda parte della narrazione, quando deve affrontare un elemento centrale nel mito di Aiace, ossia la causa della sua follia, voluta da Atena.

\section{L’originalità descrittiva di Quinto Smirneo: due affezioni oculari}

Del tutto innovativo invece rispetto ai modelli tradizionali è l'interesse di Quinto per le affezioni oftalmiche, elemento che rivela non solo i progressi medici operati in questo campo nel corso dei secoli, ma anche i moduli narrativi particolari dell'autore, dal momento che la conoscenza di questi tipi di affezioni gli permette l'introduzione di metafore ardite e la spettacolarizzazione di alcuni punti del mito.

Bisogna ricordare che la nostra conoscenza delle teorie mediche e delle terapie antiche in campo oftalmologico deriva non soltanto da trattati medici composti per la maggior parte tra il periodo alessandrino e la prima età imperiale (in particolare Erofilo, Demostene Filalete, Celso e Rufo di Efeso), ma anche dalla documentazione diretta fornita da papiri. ${ }^{33}$ Proprio questi ultimi - la maggior parte dei quali riguardano la farmacologia, ma altri anche la teoria medica oculistica e gli interventi chirurgici sull'occhio - ci permettono di comprendere come fossero definite eziologicamente e curate alcune tra le affezioni oftalmiche più diffuse. ${ }^{34}$ Proprio il bagaglio di conoscenze da noi acquisite sull'oftalmologia antica ci permetterà, come noteremo più estesamente in seguito, di capire quale fosse lo stadio delle conoscenze all'epoca di Quinto e come l'autore stesso potesse usarle inserendole nella propria opera.

Descrizioni di natura oculistica ricorrono nei Posthomerica in due punti, di cui il primo (Q.S. 1.76-85) conserva un'immagine meno precisa in quanto inserita

33 In merito allo studio dei papiri di ambito oftalmologico ritrovati in Egitto, cf. soprattutto gli studi di riferimento di Andorlini (1993) e Marganne (1994).

34 Bisogna ricordare, infatti, come notano gli studi citati alla nota precedente, il ruolo di primo piano che questo tipo di patologie aveva in un periodo storico in cui non era conosciuta la batteriologia e in una zona climatica così sfavorevole per queste affezioni come l'Egitto del tempo, quest'ultimo elemento principale causa del proliferare di documenti su rimedi e teorie mediche legati all'occhio. 
all'interno di una similitudine. Il secondo invece (12.399-415) è una narrazione abbastanza estesa e precisa di un'affezione oftalmica: si tratta, come vedremo, dell'attacco di cecità di Laocoonte indotto da Atena come punizione per la sua resistenza ad accogliere il cavallo di legno.

Il primo dei due brani, come si è notato, si trova nel primo libro, quando Priamo dopo aver perso Ettore in battaglia accoglie la regina delle Amazzoni Pentesilea, con la speranza che possa rappresentare una valida difesa per la città contro gli attacchi dei Greci. Dopo la descrizione della gioia dei Troiani nell'accogliere l'armata della regina, Quinto descrive introspettivamente i sentimenti di Priamo, il quale nonostante soffra per la perdita dei figli e la situazione in cui versa la sua città prova tuttavia sollievo nell'accogliere la donna. A questo punto l'autore, per esprimere meglio le emozioni del re troiano, inserisce una metafora di ambito medico di grande impatto. Priamo si sente come un uomo che ha sofferto moltissimo per la perdita della vista (1.76-77) e che poi, grazie all'aiuto di un dio o un medico, riesce in parte a riacquistarla (

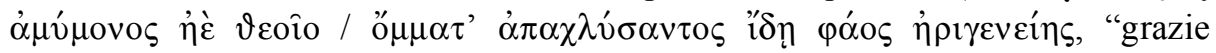
all'azione di un eccellente medico o di un dio / avendo rimosso l'oscurità dagli occhi torna a vedere la luce del giorno", 78-79) e si sente sollevato sebbene non

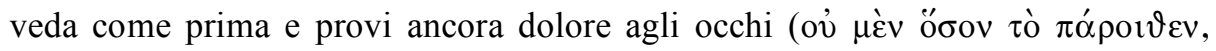

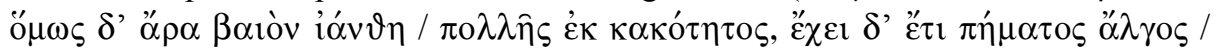

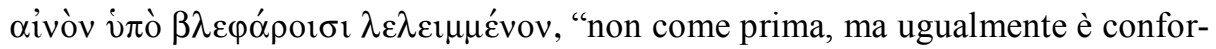
tato un poco / dopo la grande sventura, sebbene accusi ancora un atroce dolore dovuto alla disgrazia / rimasto sotto le palpebre”, 80-82). Proprio queste sensazioni - sollievo misto al continuo dolore per la perdita dei propri figli in guerra sono quelle che il re di Troia prova quando compare alla sua vista Pentesilea (82$85)$.

Come si può notare la descrizione, oltre a essere di alto impatto emotivo (tra l'altro, è sempre il vedere il verbo principale dei due momenti della similitudine, con l'uomo affetto da cecità che rivede la luce e Priamo che prova le stesse sensazioni nel guardare Pentesilea), è anche costruita con un'attenzione particolare ai sintomi diacronici e sincronici del protagonista (cecità, ripresa parziale della vista, permanenza del dolore agli occhi). Affiancati a riprese della concezione tradizionale della medicina antica - la guarigione può essere dovuta ipoteticamente all'intervento di un medico $\mathrm{o}$, come si è già notato in precedenza, a quello di un dio $^{35}$ - il brano contiene anche quelli che sembrano riferimenti più precisi alla scienza medica del tempo, soprattutto dal punto di vista lessicologico. Nonostante infatti l'immagine sia del tutto letterarizzata e inserita in un contesto estraneo alla scienza medica, sopravvivono tra le maglie della narrazione tracce di

35 Cf. in particolare la sezione precedente. 
linguaggio tecnico: il verbo $\alpha \dot{\alpha} \alpha \chi \lambda \hat{v} \sigma \alpha \nu \tau$ os (79, che nella forma $\alpha \pi \alpha \chi \lambda \hat{v} \omega$ pare essere un hapax assoluto), si avvicina molto al tecnoletto medico del tempo, dal momento che, come nota giustamente Vian (1963) 161, il sostantivo $\alpha \chi \lambda \hat{u} \varsigma$ ("mist over the eyes", LSJ s.v.), presente in letteratura già nell'epica omerica, appartiene anche al vocabolario medico oculistico. ${ }^{36}$

Diverso è il caso di Q.S. 12.399-415, in cui si narra l'attacco, causato da Atena, che porterà alla cecità Laocoonte. Proprio la natura di questa descrizione permette di comprendere quanto l'autore fosse interessato ai temi medici all'interno della sua opera, fino a trasformare le descrizioni da precise a macabre: non a caso, infatti, Quinto, decidendo di inserire un dato innovativo (o al limite decisamente poco sfruttato) della trattazione del mito, ${ }^{37}$ si dedica a fondo alla descrizione di una patologia e, svincolato dai lacci del racconto tradizionale, narra l'evento nei minimi particolari.

La punizione di Laocoonte comincia con un terremoto mandato da Atena (395-398), che decide di punire il sacerdote con la cecità (questo elemento dell'affezione mandata da un dio è l'unico, all'interno della descrizione, che pare

36 Il termine nella sua accezione tecnica indica solitamente un'affezione alla vista "as a result of ulceration" (LSJ s.v.): cf. per es. Ippocrate, Prorrheticus 2.20; Aezio, Libri medicinales 7.27 e Dioscoride, De materia medica 2.78.2 (al plurale). Più difficile individuare la patologia che ha portato all'affezione oculare da cui poi l'uomo sembra in parte essersi ripreso. Mentre van Krevelen (1964) 179 non si pronuncia a riguardo, Vian (1963) 161, sulla base soprattutto della seconda descrizione di un'affezione oftalmica di cui si parlerà in seguito, ipotizza che si possa trattare di una forma benigna di glaucoma, che quindi poteva essere in parte curabile. Sebbene l'ipotesi non sia da escludere, bisogna notare tuttavia che i dati a nostra disposizione sembrano decisamente troppo esigui per poter indicare con un buon grado di probabilità di quale malattia si trattasse, soprattutto se si considera che, come si vedrà per il passo nel dodicesimo libro, non si è certi che in quel periodo storico i diversi tipi di glaucoma fossero conosciuti in quanto tali. La similitudine inoltre si concentra sulla guarigione dall'affezione e non sui sintomi che la contraddistinguono: 1'unico elemento che può quindi aver spinto Vian (1963) 161 a ipotizzare una forma curabile di glaucoma pare il forte dolore che l'uomo accusa ancora dopo aver recuperato la vista (Q.S. 1.81-82). Sebbene però questo sintomo sia caratteristico dell'attacco di glaucoma, bisogna notare a discapito della tesi di Vian che esso sembra peculiare soprattutto di quello maligno, mentre la forma curabile si presenta spesso in modo asintomatico. In conclusione, i dati che il narratore fornisce per l'identificazione dell'affezione oculare sembrano troppo esigui per poter solo ipotizzare a quale affezione oftalmica si stia alludendo.

37 Tra le opere letterarie e mitografiche conservate, infatti, solo il brano dei Posthomerica presenta direttamente l'episodio dell'accecamento di Laocoonte da parte di Atena: in merito cf. per es. Bethe (1924). Vian (1959a) 64-68 e (1969) 81, 106 tuttavia, seguendo una proposta minoritaria all'interno della critica - cf. per es. Stubbe (1933) 39 e Kleinknecht (1944) 104 n. 3 - ha supposto a livello ipotetico che la cecità di Laocoonte fosse rappresentata almeno nel Laocoonte sofocleo. Considerata l'effettiva inesistenza di indizi testuali a favore di questa proposta - da notare che Radt ( $\left.{ }^{2} 1999\right) 331$ nell'introduzione al dramma cita Kleinknecht solo sotto la voce "ceterum" per completezza, non esplicitando la sua proposta - James (2004) 331 ritiene invece che si tratti di un'aggiunta del tutto originale da parte di Quinto. In merito alla tesi che l'accecamento di Laocoonte in questo passo e la similitudine oculistica in Q.S. 1.76-85 siano innovazioni originali di Quinto, cf. ora anche il contributo di James nel presente volume. 
riallacciarsi alla tradizione arcaica). A questo punto il narratore nota come la testa del sacerdote sia avvolta da una notte nera, preludio di quella che subirà alla vista: un atroce dolore si insinua sotto le palpebre e raggiunge il cervello attraverso le meningi (401-406). Segue - elemento centrale dell'affezione oftalmica - la descrizione dei globi oculari, che "apparivano a tratti iniettati di una grande quantità di sangue / [...], a tratti invece irrimediabilmente tendenti verso il colore grigio-

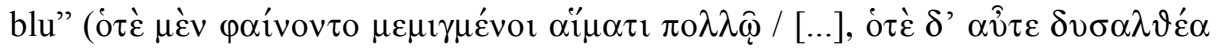
$\gamma \lambda \alpha \nu \kappa i o ́ \omega v \tau \varepsilon \varsigma, 407-408) .{ }^{38} \mathrm{~A}$ questa serie di sintomi si aggiunge una copiosa lacrimazione, paragonata all'acqua mista a neve che scende da un monte coperto di ghiacciai (409-410). ${ }^{39}$ Questa precisa relazione, quasi scientifica nel modo di procedere, dei sintomi accusati da Laocoonte è conclusa dal narratore tramite due altri dati degni di nota: il sacerdote vede doppio e urla di dolore (411-412). Nonostante tutto, Laocoonte prosegue però nell'esortare i Troiani a non fidarsi del cavallo che hanno scoperto (412-413), fino a che il narratore si sofferma sulla sua ormai completa cecità: gli occhi del sacerdote, privati della luce da Atena, "appa-

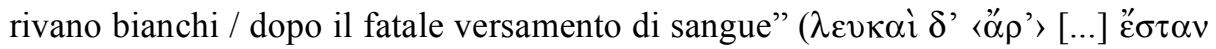

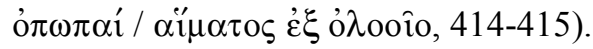

In questo caso, considerata la quantità di sintomi, si può proporre un'ipotesi sulla natura dell'affezione oftalmica che ha colpito Laocoonte. La descrizione sembra riguardare un attacco di quello che la medicina moderna (questa precisazione come si vedrà pare necessaria) indica con il termine glaucoma. ${ }^{40}$ In effetti è possibile individuare nella descrizione di Quinto i sintomi di quello che sembra, più precisamente, un glaucoma primario ad angolo chiuso: l'attacco vero e proprio è caratterizzato da un feroce dolore (qui descritto estesamente, 401-406), che a volte è legato anche a cefalea (cf. 405-406) e provoca, oltre a un edema corneale con alterazioni della pupilla (le alterazioni visibili dell'occhio sono descritte in 407-408 e poi in 414), una visione offuscata e gravemente ridotta (da notare che Laocoonte vede doppio, 411 , forse a causa della copiosa lacrimazione descritta in precedenza oppure per il procedere dell'attacco), fino al sopraggiungere della cecità (413-415).

Un problema a parte è comprendere se gli antichi testi medici avessero riconosciuto e descritto il glaucoma come una patologia oftalmica indipendente, nei termini in cui lo individua la scienza moderna. Riguardo ai papiri che conservano parti di teoria oculistica, bisogna notare che solo due di essi presentano

38 Come si noterà in seguito, la traduzione di Vian (1969) 104 "ses yeux apparaissent tantôt tout injectés de sang, tantôt au contraire vitreux, comme l'ils étaient atteints d'un glaucome incurable" sembra nell'ultima parte poco cauta, in quanto presuppone forse troppo dalla conoscenza medica antica inerente ai glaucomi.

39 In merito alla similitudine delle nevi che si sciolgono dalle cime dei monti e al suo uso in Omero e nei Posthomerica, cf. Vian (1954) 39-40.

40 Cf. van Krevelen (1964) 179, Vian (1969) 105-106 e James (2004) 331. 
sezioni, purtroppo molto frammentarie, relative al glaucoma: si tratta di PRoss. Georg. 1.20 (M-P $\left.\mathrm{P}^{3} 2343\right),{ }^{41}$ un questionario di oftalmologia, e PAshm. libr. s.n. $\left(\mathrm{M}-\mathrm{P}^{3} 2344\right),{ }^{42}$ in cui è descritto il manifestarsi del glaucoma con attenzione a eventuali concause. Nel primo testo, la parte relativa al glaucoma è quasi del tutto in lacuna e si conserva solo la risposta all'ultima domanda, ossia in che cosa il glaucoma si differenzi dalla cataratta (proprio questo rapporto sarà fondamentale per comprendere come gli antichi giudicassero la prima affezione). Da notare che, se si considera la struttura delle domande sulle altre affezioni oculari, l'ultima di esse (quella conservata anche per il glaucoma) di solito si concentra sulle differenze tra varie forme della stessa patologia: in questo caso invece il glaucoma è paragonato alla cataratta, dato fondamentale che permette di comprendere come gli antichi ritenessero queste due affezioni collegate tra loro. Più complesso il caso di PAshm., che riporta la prima descrizione critica di una $\gamma \lambda \alpha \hat{v} \kappa \omega \sigma \iota \varsigma$ (ossia di una colorazione grigio-blu dell'occhio) dovuta a una grave ferita e di esito mortale. ${ }^{43}$

Dai testi medici conservati per esteso si può invece avere una visione più chiara di come il glaucoma fosse considerato dagli antichi. Il termine $\gamma \lambda \alpha u ́ \kappa \omega \mu \alpha$, legato tecnicamente al sintomo principale del cambiamento della colorazione dell'occhio, è abbastanza antico, dal momento che compare già in Aristotele $(D e$ generatione animalium 780a 15; 17). Da questo momento in poi $\gamma \lambda \alpha \hat{v} \kappa \omega \mu \alpha$ e altri

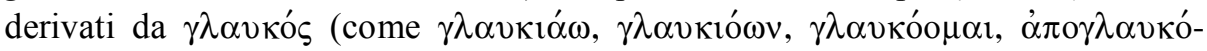

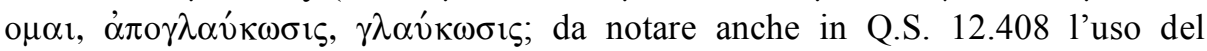
participio $\gamma \lambda \alpha v \kappa ı ́(\omega \nu \tau \varepsilon \varsigma)$ entrano nel lessico greco e latino come termini tecnici specifici indicanti le affezioni dell'occhio.

Nonostante la complessità del quadro medico antico in merito al glaucoma, si possono fissare alcuni elementi certi della concezione antica su questa affezione oculare. Come nota correttamente Marganne (1979), glaucoma, in generale, indica nell'oftalmologia antica tutta la serie di affezioni oculari che hanno come sintomo principale l'alterazione del colore della pupilla e della cornea. Nelle testimonianze più antiche - fino circa alla sistematizzazione di Rufo di Efeso che non lo considerano solo un sintomo di cattivo auspicio (come ad esempio Ippocrate nelle testimonianze citate in n. 43), il glaucoma è strettamente collegato alla cataratta, che presenta una fenomenologia esterna simile, dovuta alla parziale o completa opacità del cristallino.

41 Andorlini (1993) nr. 95; Marganne (1994) 112-132.

42 Andorlini (1993) nr. 40; Marganne (1994) 97-103.

43 Che il mutamento della colorazione dell'occhio verso il colore grigio-blu fosse ritenuta dagli antichi sintomo infausto si nota per esempio in Ippocrate, Prorrheticus 2.20; Epidemiarum liber 4.30 e Aphorismi 3.31 . 
Per meglio comprendere come gli antichi giudicassero l'affezione che chiamavano glaucoma, le diverse opinioni si possono dividere diacronicamente in tre fasi. In quella più antica, del VI-V sec. a.C. circa, il termine e i suoi derivati designano una patologia che, per via del sintomo principale del cambiamento di colorazione dell'occhio, può essere del tutto identificata dai moderni con la cataratta. Nel III sec. a.C. invece compare in un brano di Crisippo (fr. 178 von Arnim $=937$ Hülser) il termine $\dot{v} \pi$ o $\varepsilon$ ć $\mu \alpha \iota$ con il significato specifico di "avere la cataratta", insieme anche alla descrizione dell'operazione chirurgica per eliminarla. Da questo momento in poi la terminologia si bipartisce e dalle attestazioni si può chiaramente inferire come i due termini indichino ancora di base una medesima patologia, ma ne siano considerati due diverse manifestazioni: mentre infatti la cataratta è ritenuta la forma curabile, con glaucoma si indica quella maligna che porta alla perdita della vista. ${ }^{44}$ L'origine comune delle due affezioni viene in seguito eliminata da Rufo di Efeso $^{45}$ attraverso la teorizzazione di un'eziologia per il glaucoma, definito come una patologia dell'umore cristallino che cambia colore (da notare che per gli antichi è proprio il cristallino la parte principale che permette la visione nell'occhio), causa diversa quindi da quella della cataratta che si riteneva dovuta alla presenza di un umore coagulato tra iride e cristallino. Questa differenziazione delle due patologie farà scuola e verrà riassunta anche da Aezio (cf. in part. 7.52), il quale sintetizzerà le varie opinioni su natura ed eziologia del glaucoma, notando come esso possa essere dovuto al cambiamento di colore dell'umore cristallino oppure ritenuto la forma maligna della cataratta.

Marganne conclude quindi notando come gli antichi confondessero spesso nei loro trattati medici glaucoma e cataratta e consigliando quindi la vaghezza nell'interpretazione dei testi, dato che, secondo la studiosa, i medici classici non identificavano con $\gamma \lambda \alpha u ́ \kappa \omega \mu \alpha$ la patologia oftalmica moderna indicata con questo termine. Alla fine di questa argomentazione, tuttavia, la studiosa inserisce in nota, a beneficio di dubbio, il passo della cecità di Laocoonte in cui van Krevelen (e come si è visto non solo lui) vede la descrizione di un glaucoma. ${ }^{46}$ In realtà, il fatto che gli antichi non conoscessero il glaucoma esattamente nei termini con cui lo indica la medicina moderna non sembra risultare problematico per la descrizione del passo di Quinto. L'autore, infatti, riassume probabilmente quello che dagli antichi era ritenuto, senza conoscerne appieno l'eziologia, il procedere diacronico di sintomi di uno degli attacchi più violenti di cecità e che, solo grazie alla quantità di particolari, uno studioso moderno può con buon grado di probabilità identificare con un attacco acuto di glaucoma primario ad angolo chiuso.

44 Cf. per es. 1'opinione di Demostene l'oftalmologo citata in Aët. 7.53.

45 Ripreso da Oribasio, Synopsis 8.49.

46 Marganne (1979) 214 n. 79. 
In conclusione, indipendentemente dai singoli dettagli o problemi esegetici che questo passo, come quelli precedenti, inevitabilmente comporta, rimane il dato fondamentale della cospicua presenza all'interno dei Posthomerica di dettagli legati alla sfera medica. Proprio tramite l'analisi di questo aspetto è possibile fare meglio luce sul fulcro della tecnica compositiva di un autore situato di fatto alla fine del processo di evoluzione del genere epico: il suo rapporto con gli ipotesti più o meno diretti rappresentati dalla tradizione letteraria precedente e, allo stesso tempo, il suo desiderio di inserire nonostante tutto alcuni personali tasselli di innovazione e originalità all'interno di un genere così stratificato come quello dell'epica antica. 
